\title{
DATOS PRELIMINARES DE LA EXPORTACION DE MATERIA DISUELTA Y PARTICULADA EN UNA CUENCA DE ENCINAR ${ }^{1}$
}

M.J. Lledó*

A. Escarré*

\begin{abstract}
RESUMEN
En los dos últimos años se han controlado los flujos de agua y nutrientes en una cuenca de encinar de 55,5 ha. Se presentan aqui los resultados preliminares de las salidas en forma disuelta y particulada con el agua de avenamiento, y se comparan los resultados con los obtenidos en el bosque experimental de Hubbard Brook.

Las salidas en forma disuelta se han calculado a partir de datos diarios de caudal y las concentraciones de distintos iones estimadas una vez por semana. Las cantidades totales exportadas en estos dos años son 99,8 y 139,5 $\mathrm{kg}$. ha $\mathrm{ano}^{-1}$, en correspondencia con caudales anuales de 44,3 y 60,6 mm., respectivamente.

La materia particulada se ha muestreado con una trampa de sedimentos, una red y con el filtrado de agua. En cada una de estas fracciones se han medido las componentes orgánica e inorgánica. La salida total en forma particulada es de 0,98 kg. ha el primer año y de sólo $0,52 \mathrm{~kg}$. ha el segundo. Estos valores son muy pequeños si se comparan con los correspondientes a otros ecosistemas maduros y conservadores en su exportación erosiva.

La pequeña pérdida de materia particulada se relaciona con el mínimo caudal de avenamiento anual, sólo el $14,2 \%$ de las entradas por precipitación, pero otros mecanismos que tienden a minimizar la erosionabilidad resultan también de importancia.
\end{abstract}

1. Este estudio ha sido subvencionado en parte por la CAICYT (proyecto $n .^{\circ} 770$ ) y también con fondos del proyecto LUCDEME.

* Departamento de Biología. Facultad de Ciencias, Apartado 99, Alicante. 


\section{M.J. LLEDO, A. ESCARRE}

\section{SUMMARY}

In the last two years, a 55,5 ha watershed, covered by an evergreen-oak forest has been monitored for hydrological and nutrient fluxes. Preliminary results on dissolved and particulate outputs with the runoff are presented here, and compared with data obtained in the Hubbard Brook Experimental Forest.

Dissolved output has been calculated with daily water flow data and weekly concentration values for the different ions. The amounts exported in the two years are 99,8 and $139,5 \mathrm{~kg}$. ha $\mathrm{h}^{-1} \mathrm{yr}^{-1}$, corresponding to yearly water flows of 44,3 and $60,3 \mathrm{~mm}$.

Particulate matter has been sampled with a trap, a net and filtering water. In each fraction the organic and inorganic components has been measured. The particulate output is $0,98 \mathrm{~kg}^{\mathrm{h}} \mathrm{ha^{-1 }}$ in the first year, and only 0,52 $\mathrm{kg} . \mathrm{ha}^{-1}$ in the second. This values are minimal if compared with export data from other mature ecosystems very conservative in your particulate exportation.

The little loss of particulate matter is related to the minimal yearly water runoff, only the $14,2 \%$ of the water input in precipitation, but other mechanismes minimizing erodibility are also important.

El estudio biogeoquímico de ecosistemas delimitados en pequeñas cuencas con substrato impermeable ha permitido una mejor comprensión de los procesos que generan y regulan los flujos de nutrientes entre los diversos compartimentos del sistema (Likens et al. 1977; Bormann et al. 1979), y alguna generalización sobre la capacidad de retención de nutrientes en relación con la sucesión (Vitousek \& Reiners 1975; Vitousek 1983).

Además de en el área pionera de Hubbard Brook, la metodología del control de pequeñas cuencas se está siguiendo en diversas zonas del planeta, muy especialmente en las que tienen mayor interés forestal. Para la región mediterránea, en la que no existen precedentes de este enfoque, el presente trabajo pretende aportar algunos de los datos preliminares sobre la exportación de materiales en forma disuelta y particulada desde una cuenca con bosque esclerófilo, y establecer las primeras comparaciones con los resultados del bosque mixto de Hubbard Brook obtenidos durante un mayor número de años de estudio, con procedimientos de muestreo y análisis que se ha procurado seguir con la mayor fidelidad posible.

\section{Area de estudio}

El presente trabajo se ha realizado en el Barranc de l'Avic que es una cuenca impermeable con una superficie de 55,5 has., situada en el Monte 


\section{EXPORTACION DE MATERIA EN UNA CUENCA DE ENCINAR}

Poblet (Serra de Prades; Tarragona). La vegetación que le cubre es un encinar, explotado antiguamente para carboneo, con numerosos rebrotes de Quercus ilex como especie dominantes, Arbutus unedo y Phillyrea media. El suelo es de tipo Xerochrept y la roca madre consta de pizarras y areniscas pizarrosas del Carbonífero. Una referencia más detallada del lugar, así como de las instalaciones de muestreo se pueden encontrar en Escarré et al. (1982).

\section{Metodología}

Para controlar las salidas del ecosistema se ha construido una estación de aforo, y poco antes de la misma se recogen muestras para análisis del material disuelto que se verifican mediante Cromatografía Iónica, Espectrofotometría de Absorción Atómica y otros métodos colorimétricos.

Para la recolección de materia particulada se sigue la metodología descrita en Bormann et al. (1969 y 1974), aunque con alguna modificación.

$\mathrm{El}$ transporte de los materiales en una cuenca se puede realizar en forma de suspensión, saltación y acarreo o arrastre, aunque generalmente la saltación se engloba con el acarreo (López Cadenas \& Blanco, 1968).

La materia particulada de arrastre se recoge en una trampa por medio del vaciado de bandejas con distintas mallas dispuestas en su interior una vez al mes aproximadamente. La parte orgánica de este material está formado por ramas, ramillas, hojas, e invertebrados, cuya estructura está prácticamente intacta, y que por tanto se puede separar sin posibilidad de error. La parte inorgánica gruesa está compuesta por fragmentos de pizarra que raramente supera los tres centímetros de diámetro medio. Existe una tercera parte formada por material fino en la que la separación en estas dos fracciones es difícil. Para determinar la parte orgánica e inorgánica del material más fino se ha procedido a calcular provisionalmente el porcentaje medio de cada fracción en 6 muestras y se ha aplicado dicho valor en todos los muestreos.

La materia particulada en suspensión se recoge aproximadamente una vez a la semana en dos tamaños: mayor de $1 \mathrm{~mm}$., mediante una red que está puesta a la salida de la trampa continuamente, y entre $1 \mathrm{~mm}$. y 0,45 micras, mediante el filtrado de unos 10 litros cada semana. En la red la separación de orgánico e inorgánico se realiza a mano, mientras que el procedimiento seguido en el filtro ha sido calcinación de 4 de ellos, en espera de tener mejores resultados del análisis químico de los materiales recolectados. 


\section{Resultados y discusión}

La exportación de materia disuelta y particulada desde la cuenca de l'Avic, así como la salida de agua aparecen en la tabla $n .^{\circ} 1$, en la que los datos de caudal y material disuelto se ha hecho corresponder a los períodos de recolección de materiales particulados que aparecen indicados.

En el primer año con un avenamiento de 44,3 mm. se exportan 99,8 kg. $\mathrm{ha}^{-1}$ en forma disuelta y $0,98 \mathrm{~kg}$. ha ${ }^{-1}$ en forma particulada, mientras que al segundo año con un caudal de $60,3 \mathrm{~mm}$. le corresponden $139,5 \mathrm{~kg}$. ha $\mathrm{ha}^{-1}$ en forma disuelta y 0,52 en forma particulada.

Con los datos de esta tabla se han investigado las relaciones entre el caudal y las salidas de sustancias en forma disuelta y particulada, con lo que se ha puesto de manifiesto que para la primera el ajuste de la relación lineal es altamente significativo, y mejora muy poco en la forma exponencial; sin embargo para la materia particulada aunque el ajuste lineal es significativo, mejora considerablemente con la transformación logarítmica (fig. 1).

El máximo caudal se produce en el período entre el 23 de noviembre y el 19 de diciembre de 1982 con un avenamiento de $28,2 \mathrm{~mm}$. En este período se exporta la mayor cantidad de sustancias disueltas de todo el estudio $61,2 \mathrm{~kg}$. ha ${ }^{-1}$ y sin embargo no ocurre lo mismo en la materia particulada. Para esta última el período con mayor exportación es el comprendido entre el 20 de julio y el 28 de octubre de $1982\left(0,58 \mathrm{~kg}\right.$. ha- ${ }^{-1}$ donde el avenamiento es tan sólo de $14,2 \mathrm{~mm}$.

Las salidas anuales de cationes se relacionan bien linealmente con el caudal (Likens et al., 1977), lo que se hace extensible también a aniones si se consideran salidas y caudales mensuales (Terradas \& Escarré 1983).

El modelo más general (Bormann et al. 1969) supone que la concentración es prácticamente constante y por tanto independiente del caudal. Sin embargo tanto en Hubbard Brook como en l'Avic se pueden constatar ciertas diferencias en las concentraciones ponderadas referentes a las distintas estaciones.

En cualquiera de las dos zonas de estudio la razón entre los valores de concentración máximos y mínimos sólo es superior a 2 en los compuestos de nitrógeno, pero en los demás iones raramente excede el valor de 1,5. 


\section{EXPORTACION DE MATERIA EN UNA CUENCA DE ENCINAR}

Tabla 1. Valores de avenamiento, expresados en mm., salida de sustancias disueltas, expresados en $\mathrm{kg}$. ha $\mathrm{h}^{-1}$ y salida de materia particulada, expresado en $\mathrm{g} \mathrm{ha}^{-1}$, así como de los porcentajes en que contribuye cada período considerado al total en las tres salidas de la cuenca de l'Avic.

\begin{tabular}{|c|c|c|c|c|c|c|c|}
\hline \multirow[t]{2}{*}{ Período } & \multirow[t]{2}{*}{ Días } & \multicolumn{2}{|c|}{ Avenamiento } & \multicolumn{2}{|c|}{$\begin{array}{c}\text { Sustancias } \\
\text { disueltas }\end{array}$} & \multicolumn{2}{|c|}{$\begin{array}{c}\text { Materia } \\
\text { particulada }\end{array}$} \\
\hline & & $\mathrm{mm}$ & $\%$ & $\mathrm{~kg} \mathrm{ha}^{-1}$ & $\%$ & $\mathrm{gr} \mathrm{ha}^{-1}$ & $\%$ \\
\hline 06 Dic. $81-19$ Dic. 81 & 14 & 0,15 & 0,14 & 0,52 & 0,22 & 5,26 & 0,35 \\
\hline 20 Dic. $81-03$ En. 82 & 15 & 0,28 & 0,27 & 0,91 & 0,38 & 6,04 & 0,40 \\
\hline 04 En. 82 - 19 En. 82 & 16 & 0,31 & 0,29 & 1,01 & 0,42 & 11,03 & 0,73 \\
\hline 20 En. $82-26$ Feb. 82 & 38 & 5,98 & 5,71 & 15,67 & 6,55 & 138,91 & 9,18 \\
\hline 27 Feb. 82 - 28 Mar. 82 & 30 & 3,48 & 3,32 & 8,66 & 3,62 & 15,88 & 1,05 \\
\hline 29 Mar. 82 - 22 Abr. 82 & 25 & 3,06 & 2,92 & 7,72 & 3,22 & 20,73 & 1,37 \\
\hline 23 Abr. 82 - 23 May. 82 & 31 & 1,85 & 1,76 & 4,45 & 1,85 & 8,49 & 0,56 \\
\hline 24 May. 82 - 17 Jun. 82 & 25 & 1,34 & 1,28 & 3,85 & 1,61 & 12,41 & 0,82 \\
\hline 18 Jun. 82 - 19 Jul. 82 & 32 & 0,19 & 0,18 & 0,69 & 0,29 & 2,02 & 0,13 \\
\hline 20 Jul. 82 - 28 Oct. 82 & 101 & 14,17 & 13,17 & 26,46 & 11,05 & 580,50 & 38,37 \\
\hline 29 Oct. 82 - 22 Oct. 82 & 25 & 13,56 & 12,95 & 29,94 & 12,50 & 188,07 & 12,44 \\
\hline 23 Nov. 82 - 19 Dic. 82 & 27 & 28,57 & 26,99 & 61,21 & 25,57 & 344,11 & 22,75 \\
\hline 20 Dic. $82-12$ En. 83 & 24 & 8,36 & 7,98 & 22,65 & 9,46 & 43,04 & 2,84 \\
\hline 13En. $83-21$ Feb. 83 & 40 & 8,41 & 8,03 & 18,80 & 7,85 & 21,82 & 1,44 \\
\hline 22 Feb. $83-19$ Abr. 83 & 67 & 6,57 & 6,27 & 15,62 & 6,52 & 22,02 & 1,46 \\
\hline 20 Abr. 83 - 18 May. 83 & 29 & 1,56 & 1,49 & 3,74 & 1,56 & 7,01 & 0,46 \\
\hline 19 May. 83 - 14 Jun. 83 & 27 & 9,58 & 0,55 & 1,32 & 0,55 & 5,58 & 0,38 \\
\hline 15 Jun. 83 - 31 Jul. 83 & 47 & 0,03 & 0,03 & 0,10 & 0,04 & 1,34 & 0,09 \\
\hline $01 \mathrm{Ag} .83-27 \mathrm{Ag} .83$ & 27 & 0,04 & 0,04 & 0,13 & 0,05 & 2,17 & 0,14 \\
\hline $28 \mathrm{Ag} .83-01$ Nov. 83 & 66 & 0,88 & 0,84 & 2,48 & 1,03 & 4,08 & 0,27 \\
\hline 02 Nov. $83-06$ Dic. 83 & 35 & 5,68 & 5,42 & 13,47 & 5,64 & 72,21 & 4,77 \\
\hline
\end{tabular}

Tabla 2. Valores de concentraciones medias por estaciones en los dos años de estudio de l'Avic, de los distintos elementos que se analizan también en Hubbard Brook. Expresados en mg $\mathrm{L}_{-\mathrm{I}}$.

\begin{tabular}{|l|r|r|r|r|}
\cline { 2 - 5 } \multicolumn{1}{c|}{} & Otoño & Invierno & Primavera & Verano \\
\hline $\mathrm{F}^{-}$ & 0,25 & 0,22 & 0,19 & 0,16 \\
$\mathrm{Cl}^{-}$ & 9,38 & 11,29 & 8,84 & 10,02 \\
$\mathrm{SO}^{+}+$ & 29,21 & 35,45 & 31,69 & 39,30 \\
$\mathrm{Ca}^{+}$ & 51,70 & 41,40 & 27,87 & 52,85 \\
$\mathrm{Mg}_{+}^{++}$ & 8,40 & 7,76 & 8,16 & 10,86 \\
$\mathrm{Nq}^{+}$ & 13,24 & 12,52 & 13,28 & 14,37 \\
$\mathrm{~K}^{+}$ & 1,95 & 1,83 & 1,59 & 1,85 \\
$\mathrm{NH}_{4}^{+}$ & 0,04 & 0,01 & 0,07 & 0,05 \\
\hline
\end{tabular}


En la tabla 2 aparecen las concentraciones de los elementos que han sido analizados tanto en l'Avic como en Hubbard Brook. Si se comparan los coeficientes de variación de los datos de esta tabla con los correspondientes a Hubbard Brook se observan valores bastante superiores para calcio y sulfato en l'Avic, donde son iones mayoritarios y para el potasio en Hubbard Brook probablemente como un reflejo del carácter predominantemente caducifolio del bosque.

La distribución del total de sustancias disueltas en los correspondientes iones durante los dos años de estudio viene en la tabla 3. Como aniones mayoritarios aparecen el bicarbonato y el sulfato y como catión el calcio. Hay que destacar que no está contabilizada la sílice disuelta, que podría suponer cantidades importantes de exportación, ni los fosfatos y nitratos que por el contrario resultan ser cantidades inapreciables a tenor de las concentraciones observadas en algunos de los meses de estudio (Escarré et al. 1984).

Tabla 3. Valores totales de la exportación de los distintos iones analizados, en los dos años en que se ha estudiado la materia particulada, expresados en $\mathrm{kg}$. $\mathrm{ha}^{-1}$

\begin{tabular}{|r|c|c|c|c|c|c|c|c|c|}
\hline Período & $\mathrm{F}^{-}$ & $\mathrm{Cl}^{-}$ & $\mathrm{SO}_{4}^{=}$ & $\mathrm{CO}_{3} \mathrm{H}^{-}$ & $\mathrm{Ca}^{++}$ & $\mathrm{Mg}^{++}$ & $\mathrm{Na}^{+}$ & $\mathrm{K}^{+}$ & $\mathrm{NH}_{4}^{+}$ \\
\hline $\begin{array}{r}6-12-81 \\
22-11-82\end{array}$ & 0,22 & 4,10 & 12,77 & 57,54 & 14,93 & 3,15 & 5,26 & 0,84 & 0,19 \\
\hline $\begin{array}{r}23-11-82 \\
5-12-83\end{array}$ & 0,12 & 5,59 & 24,86 & 75,39 & 22,26 & 4,48 & 6,32 & 0,89 & 0,11 \\
\hline
\end{tabular}

Para que los valores de exportación de material disuelto sean comparables con el valor medio de 8 años de estudio en Hubbard Brook, se puede corregir el efecto debido al caudal, dando la exportación en $\mathrm{kg}$. ha ${ }^{-1} \mathrm{año}^{-1}$ $\mathrm{mm}^{-1}$ que para l'Avic da valores de 2,25 y 2,30 respectivamente en dos años y para Hubbard Brook $0,13 \mathrm{~kg}$. ha ${ }^{-1} \mathrm{año}^{-1} \mathrm{~mm}^{-1}$. Como se ve los resultados para l'Avic son poco menos de veinte veces el valor de Hubbard Brook que precisamente tiene un caudal medio casi 20 veces superior a los valores de l'Avic. Esta compensación hace que la exportación de sustancias disueltas sea similar en ambos ecosistemas pese a la gran diferencia de caudal.

La relación entre la materia disuelta (excluida la sílice) y materia particulada tiene un valor medio en Hubbard Brook para 5 años de estudio de 3,2 , alcanzando en algún período valores próximos a 10. En l'Avic los valores para el primer y segundo año de esta misma relación son 101 y 265, cifras extraordinariamente elevadas como consecuencia de que los valores de 


\section{EXPORTACION DE MATERIA EN UNA CUENCA DE ENCINAR}

material particulado son en l'Avic inferiores a $1 \mathrm{~kg}$. ha ${ }^{-1} \mathrm{año}^{-1}$ mientras que el valor medio obtenido en Hubbard Brook es de $33 \mathrm{~kg}$. ha- ${ }^{-1}$. Si se expresa la relación de la materia particulada en $\mathrm{kg}$. ha $\mathrm{a}^{-1} \mathrm{año}^{-1} \mathrm{~mm}^{-1}$, la estima para Hubbard Brook es de 0,039 mientras que para l'Avic son 0,022 y $0,009 \mathrm{~kg}$. $\mathrm{ha}^{-1} \mathrm{año}^{-1} \mathrm{~mm}^{-1}$ respectivamente, valores menores pero mucho más comparables que los cocientes antes mencionados.

La escasa exportación de materia particulada tiene que ver con que en el año hidrológico mejor definido en l'Avic el avenamiento total es de sólo $81,6 \mathrm{~mm}$., unas 10 veces menor que el medio para Hubbard Brook y que representa sólo el $14,2 \%$ de las entradas por precipitación. El elevado porcentaje que corresponde entonces a la evapotranspiración $(85,8 \%)$ contribuye a minimizar las posibilidades de exportación en los flujos acuosos.

Entre los mecanismos citados por Bormann et al. (1974) como más importantes en el control de la erosionabilidad, son sobre todo de destacar en l'Avic la protección de la cubierta vegetal, el hecho de que el recorrido del arroyo sea subalveo en su casi totalidad para los valores de caudal más usuales y también la formación de depósitos de materiales orgánicos en algunas porciones del recorrido superficial del arroyo.

La relación entre la cuantía e intensidad de la precipitación y la cantidad de materia particulada exportada en el período pone de manifiesto salidas importantes para períodos con precipitaciones totales en general superiores a los $100 \mathrm{~mm}$. en las que se han registrado intensidades de lluvia superiores a $\operatorname{los} 3 \mathrm{~L} \mathrm{~h}^{-1}$, si bien se tiene registrada una tormenta de agosto de 1983 de 63,5 L en dos horas y media que debido al extraordinario déficit hídrico del suelo, apenas originó avenamiento y un mínimo arrastre de material particulado.

Un aspecto de difícil medida dada la especial dinámica de exportación en materia particulada, es el de las tasas instantáneas de descargas de materiales. En Hubbard Brook se ha estimado que el $86 \%$ de la materia particulada se pierde en tan sólo el 1,6\% del tiempo y el $23 \%$ del volumen de agua, pero aceptando una cierta distribución del material almacenado en la presa cuya recolección es entre trimestral y anual. En una tormenta en la que la recolección de material particulado se hizo muy poco después de producirse, se ha podido ver que el $38,8 \%$ del total exportado en el año se produjo como máximo en el $2,7 \%$ del tiempo y el $13,4 \%$ del caudal anual del Barranc de l'Avic.

Las diferentes fracciones de materia particulada recolectadas con la trampa, la red y el filtro se dan para los dos años en la tabla 4. Las diferencias entre los dos años son apreciables, y mientras que en el primero dominan los materiales recolectados en la trampa, en el segundo es la recolección por filtrado la que aporta mayor peso de material. 
Tabla 4. Valores de materia particulada desglosados en sus componentes orgánica e inorgánica, para las tres fracciones de recolección y los dos años de estudio, expresados en g. $\mathrm{ha}^{-1}(\mathrm{O}$, orgánico; I, inorgánico).

\begin{tabular}{|l|c|c|c|c|c|c|}
\cline { 2 - 6 } \multicolumn{1}{c|}{} & $\mathrm{O}$ & $\mathrm{I}$ & $\mathrm{O}$ & $\mathrm{I}$ & $\mathrm{O}$ & $\mathrm{I}$ \\
\hline 1. ${ }^{\mathrm{er}}$ año & 287,9 & 314,7 & 102,6 & 0,4 & 64,3 & 219,5 \\
$2 .^{\circ}$ año & 28,3 & 18,1 & 7,8 & 0,1 & 136,1 & 333,0 \\
\hline
\end{tabular}

Cuando se consideran los valores correspondientes a los distintos períodos, éstos resultan predecibles en muy distinto grado a partir del caudal, y así, mientras que los datos obtenidos con la filtración presentan elevados valores del coeficiente de correlación tanto en la regresión lineal como en la logarítmica $(0,92$ y 0,96$)$, las fracciones correspondientes a la trampa y la red sólo admiten un ajuste logarítmico y con menores valores del coeficiente de correlación $(0,57$ y 0,61$)$.

Un aspecto de especial interés en el que los datos son todavía muy preliminares es el de la proporción de componentes orgánica e inorgánica en cada una de las fracciones de recolección de materia particulada. De forma algo provisional se tiene una estima de la proporción de $57,4 \%$ de materia orgánica para la fracción de arrastre, del $97 \%$ para lo recogido en la red y del 77,3\% para los filtros en los que el número de mediciones ha sido especialmente pequeño. Los valores correspondientes en Hubbard Brook son $40,9 \%$ de material orgánico para la presa, $97,5 \%$ en la red y sólo $49 \%$ en el filtro, aunque este último valor medio tiene menos significado al haberse demostrado un decrecimiento asintótico del porcentaje orgánico con el caudal.

De los componentes orgánico e inorgánico para cada una de las fracciones y en lo referente a su posible predicción a partir del caudal cabe señalar que las fracciones orgánica e inorgánica de los filtros son las más predecibles, la inorgánica de la trampa y la orgánica de la red se pueden prever de forma aceptable, mientras que la orgónica de la trampa y la escasa fracción inorgánica de red presentan valores del coeficiente de correlación, en regresión logarítmica, muy próximos al límite mínimo de significación. Comparadas en su conjunto, el ajuste correspondiente a materia inorgánica es mejor que el de la materia orgánica. 


\section{Bibliografía}

BORMANN, F.H.; LIKENS, G.E. \& EATON, J.S., 1969. Biotic regulation of particulate and solution losses from a forested ecosystem. BioScience 19:600-610.

BORMANN, F.H.; LIKENS, G.E.; SICCAMA, T.G.; PIERCE, R.S.; \& EATON, J.S., 1974. The export of nutrients and recovery of stable conditions deforestation at Hubbard Brook. Ecol. Monogr. 44: 255-277.

BORMANN, F.H. \& LIKENS, G.E., 1979. Pattern and process in a forested ecosystem. Springer-Verlag, 253 pp.

ESCARRE, A.; BELLOT, J.; LLEDO, M.J.; HERVAS, J.; PUJALTE, E. \& GARRIDO, P., 1982. Balance de nutrientes en pequeñas cuencas de encinar. I. Selección de la zona de estudio e instalación de instrumentos para la recogida de datos. Mediterranea Ser. Biol. 6: 141-165.

ESCARRE, A.; LLEDO, M.J.; BELLOT, J.; SANCHEZ, J.R.; ESCLAPES, A.; CLEMENTE, A. \& ROVIRA, A., 1984. Compartimentos y flujos bioquímicos en un encinar, distinto predominio de factores físicos y biológicos en su control. IV Conferencia Cientifica de Ciencias Naturales, Universidad de La Habana.

LIKENS, G.E.; BORMANN, F.H.; PIERCE, R.S.; EATON, J.S. \& JOHNSON, N.M., 1977. Biogeochemistry of a forested ecosystem. Springer-Verlag, $146 \mathrm{pp}$.

LOPEZ CADENAS, F. \& BLANCO, M., 1968. Aspectos cualitativos y cuantitativos de la erosión hídrica y del transporte y depósito de materiales. Ministerio de agricultura, $190 \mathrm{pp}$. Barcelona.

TERRADAS, J. \& ESCARRE, A. (dirs) 1983. Ciclos de nutrientes en ecosistemas terrestres (bosques) estudiados en cuencas de pequeña extensión. Memoria final del proyecto de investigación cooperativa 0370, 1979-1983, 4 vols. Bellasterra (Barcelona).

VITOUSEK, P.M., 1983. A general theory of forest nutrient dynamics. ESF Workshop, Uppsala.

VITOUSEK, P.M. \& REINERS, W.A., 1975. Ecosystem succesion and nutrient retention: A hypothesis. BioScience 25: 376-381. 
